\title{
Virtual Vehicle Kinematics and Compliance Test Rig
}

\author{
Peter Sundström ${ }^{1} \quad$ Maria Henningsson ${ }^{1} \quad$ Xabier Carrera Akutain $^{2} \quad$ Yutaka Hirano $^{3} \quad$ Alejandro Ocariz $^{2}$ \\ Hiroo Iida $^{3}$ Naoki Aikawa ${ }^{3}$ Johan Andreasson $^{4}$ \\ ${ }^{1}$ Modelon AB, Sweden, \{peter. sundstrom, maria.henningsson\} @modelon. com \\ ${ }^{2}$ Toyota Motor Europe, Belgium, \{Alejandro.Ocariz, Xabier. Carrera.Akutain\} @toyota-europe.com \\ ${ }^{3}$ Toyota Motor Corporation, Japan, \{hiroo_iida, naoki_aikawa, yutaka_hirano\}@mail.toyota.co.jp \\ Modelon KK, Japan, johan. andreasson@modelon. com
}

\begin{abstract}
This paper presents a virtual kinematics \& compliance (K\&C) test rig, also known as a Suspension Parameter Measurement Machine, SPMM. The focus is to explain the requirements and implementation of the rig model is built to be a virtual equivalent to the physical test rig, capable of reading the same input and producing the same output.

The virtual test rig is implemented as a Modelica model that is plug compatible with any vehicle model using the standard interface from the Modelica Vehicle Dynamics Library. The operation of the virtual test rig is done from a scripting environment that executes a cosimulation FMU.

An example test case is also shown where results from a virtual test is compared with the corresponding run on the physical test rig.
\end{abstract}

Keywords: virtual testing, kinematics and compliance $(K \& C), F M I$, vehicle dynamics

\section{Introduction}

A kinematics and compliance $(\mathrm{K} \& \mathrm{C})$ test rig, Figure 1, is one of the most common way to benchmark and fingerprint cars today. A model which can reproduce the excitations and generate output in exactly the same fashion as an established physical rig presents several advantages:

The most obvious one is the time reduction in the execution of the complex tasks of the $K \& C$ test rig, measurable in terms of orders of magnitude. Carefully considering the testing efficiency, the level of confidence and relative importance of each, the K\&C test rig owner can choose to allocate the testing resources more selectively. For instance, a batch of lower priority tests could be skipped and replaced with simulation, provided the data available and/or measured from the fundamental tests is enough to generate a high fidelity base vehicle model.

Most of the OEMs are pursuing a strategy of creating multiple derivatives of the same vehicle platform which in turn generate a need for extensive testing. Again, provided a reliable base model, the $\mathrm{K} \& \mathrm{C}$ simulation of

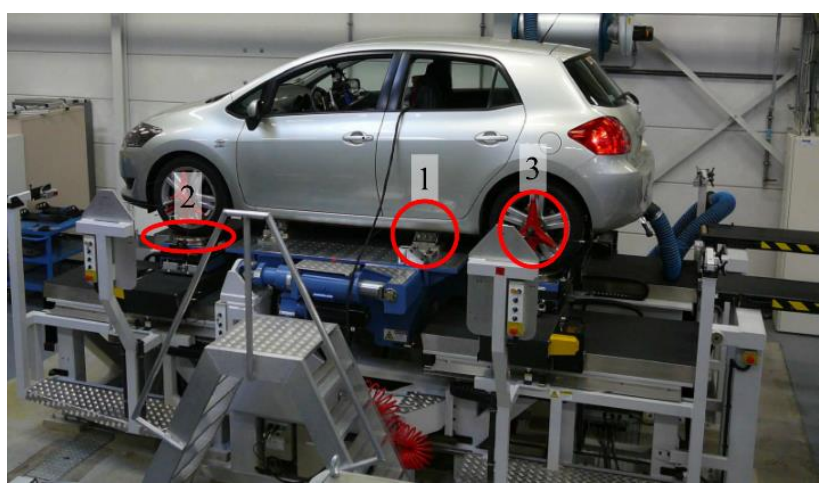

Figure 1. Kinematics and Compliance $(\mathrm{K} \& \mathrm{C})$ rig in operation at Toyota. The vehicle body is clamped (1) so that the rig can induce roll, pitch and bounce motion of the body. Under each wheel, there is a pad (2) that can move and rotate to induce tire forces and moments. On each wheel, sensors (3) that measure wheel center motion are attached.

these derivatives present a major time and cost saving in terms of vehicle availability and testing resources can be significant.

Very connected to this, the utilization of the virtual environment makes as well possible to perform system identification in order to achieve higher fidelity of the model to study, especially important if there's a significant amount of unknown parameters on the initial model. A more precise parametrization can be used for the optimization of the base vehicle model and all its derivatives according to the desired elasto-kinematic targets. Similarly, quick and inexpensive virtual sensitivity analysis of the model can help to quantify the robustness of the base performance and to study potential performance improvements instead of replacing chassis components with limited criteria.

The ownership of complete $\mathrm{K} \& \mathrm{C}$ test rigs is restricted to big or specialized corporations. On top of the reasons exposed above, sharing a common simulation rig can enhance the cooperation significantly between different industry or academia partners for every effort on chassis optimization. In this joint effort the authors have targeted a full replication of the excitations and 
workflow of an existing $\mathrm{K} \& \mathrm{C}$ test rig with the highest fidelity possible, in order to make it a useful tool in the vehicle development cycles. Additionally, to make it deployable throughout the organization, the following requirements are identified:

1. The operation of the virtual test rig should mimic the real one, especially it should be compatible with the data formats used

2. It should be possible to operate the virtual test rig without knowing the model and/or how to operate the modeling environment.

Figure 2 gives an overview of the developed toolchain and its connection to the physical test rig and related tools. The core of the toolchain is a Modelica model that is compiled as a co-simulation FMU that is able to pull parameter data from a data base (Andreasson, J. et. al., 2016). This is then executed from the deployment environment according to the test specification. The input to and the output from the virtual test rig are compatible with the corresponding real test rig signals. This allow data to be exchanged at (1) and (2). Additionally, there is a tuner that can be used to fit parameters to measurement (or other simulation) data.

The deployment environment is MATLAB with FMI support (FMI Toolbox for MATLAB, 2016) and it is chosen since the platform is widely available for the target engineers to meet requirement 2 listed above. However, the deployment could just as well be carried out in for example Python (Andersson C., 2013), (Nilsson, T., 2013) and (pyFMI, 2016) or MS Excel (FMI Add-in for Excel, 2016).

\section{Vehicle Test Rig Model}

Testing a vehicle in a test rig as opposed to on a test track is beneficial for a number of reasons. The rig can accurately reproduce load cases while iterating on suspension setups or part changes which is critical when evaluating how these changes affect vehicle performance. Furthermore, the rig can produce load cases which are difficult, dangerous or even impossible to achieve in driving scenarios.

Evaluating the kinematics and compliance of vehicle suspensions gives good insight into how the wheel and tire will move relative to the chassis and thus also what forces will be generated for different vehicle states. Essentially, the $\mathrm{K} \& \mathrm{C}$ curves for a chassis is the fingerprint of the suspension which can readily be compared between different setups or competitor vehicles to predict performance.

The results from a K\&C test are usually expressed in the form of curves or gradients. The curves describe how for example the toe angle changes with wheel travel, known as bump steer. These curves give vehicle dynamics engineers a good idea of the performance of the vehicle and are important in defining and evaluating requirements.

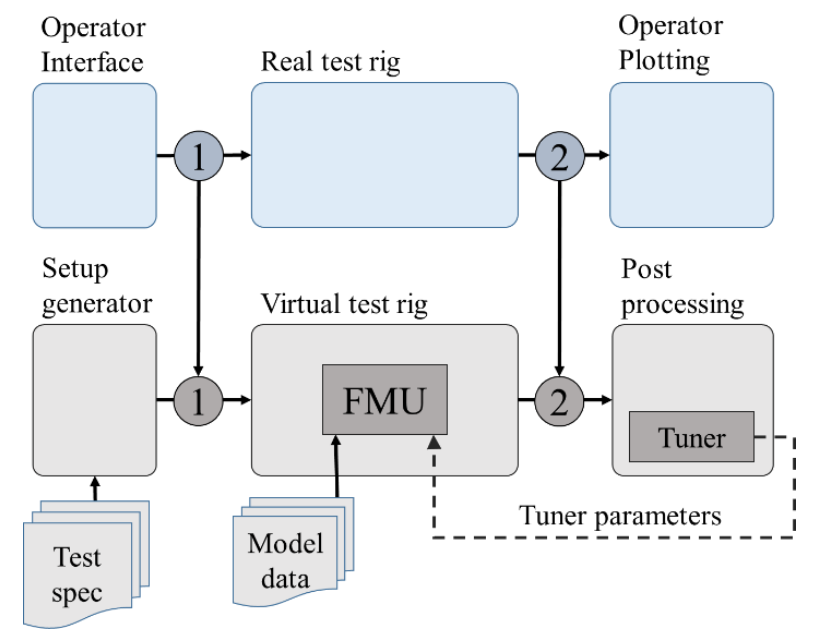

Figure 2. Toolchain overview. The real test rig receives information on execution (1) from the operator interface and then output measurement results (2) that is used for plotting. The virtual test rig is designed to be able to read and write compatible data.

The main independent quantity for $\mathrm{K} \& \mathrm{C}$ curves is normally vertical wheel travel. For a non-steered, independent suspension, the kinematics can be completely defined as a function of wheel travel. For steered suspensions, steering wheel angle or steering rack displacement is normally used as the second independent quantity. Suspension where there is a coupling between left and right sides (dependent or semi-dependent suspensions) need the wheel travel of the opposite wheel as an additional independent quantity.

Toe/steer and camber/inclination angles are key dependent quantities to study as they greatly affect the tire force generation. The change in lateral and longitudinal position are also important as this gives additional information about the momentary center of rotation of the suspension.

The compliance properties of the suspension are key to vehicle handling since these affect how the wheel angles and position changes with load, e.g. during cornering or braking. While typically measured in rigs capable of higher frequency excitation than described here, compliance also has large effect on how noise and vibration is transmitted through the chassis. Details of suspension kinematics and compliance effect on vehicle performance can be found in standard literature, see e.g. (Bastow, 2004) or (Milliken, 1995).

\subsection{Physical Test Rig}

The virtual test rig is based on an off-the-shelf physical test rig capable of exciting the vehicle chassis as well as the in plane motion of the wheels (Anthony Best Dynamics Ltd, 2014).

Before a test is performed, the vehicle is driven onto the test rig and the body is clamped to the rig table. The 
table is able to move vertically as well as roll and pitch the vehicle body relative to the ground plane. The wheels of the vehicle are positioned on wheel pads which can move in the ground plane and rotate around the vertical axis to generate planar forces and torque at ground level. By changing the wheel clamping method, forces and moments can also be applied in all directions at the wheel hubs.

The rig can perform many different test cases including the basic roll, pitch and heave movements of the chassis as well as force sweeps at the tires and/or hubs at varying ground heights.

\subsection{Virtual Test Rig}

The virtual test rig is designed to mimic the functionality of the physical test rig and to allow them to be operated in a consistent way by sharing parameterization for configuration as well as data formats. This has some important implications on the test rig model implementation. Figure 3 shows a screenshot of a vehicle model on the virtual test rig. The large yellow plate corresponds to the table to which the body is clamped and the four circular pads are used to actuate the wheels.

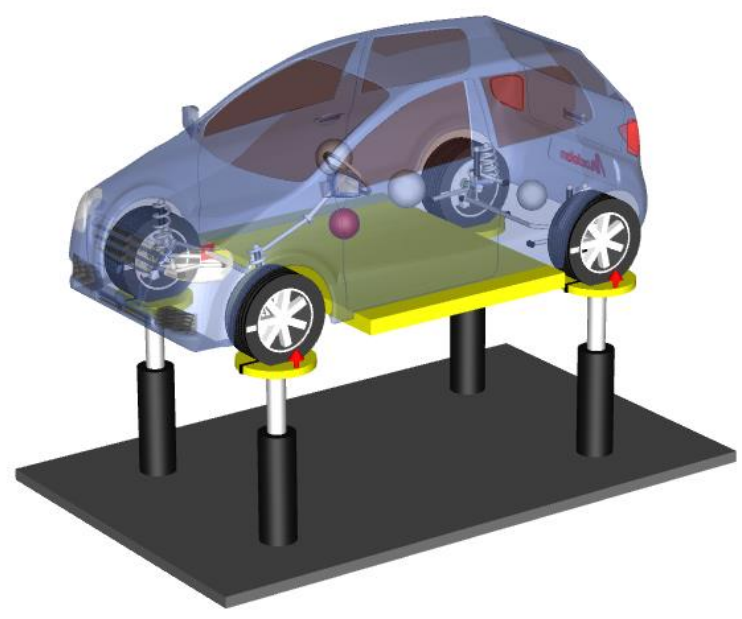

Figure 3. Animation screenshot of a vehicle in the virtual test rig. The vehicle is equipped with an independent steerable front suspension of MacPherson type and a dependent rear suspension of twist beam type.

Since the model is deployed as FMUs in a non-Modelica environment, care is taken to design the virtual test rig so that it does not have to be recompiled for the different operating modes. Additionally, since typical test procedure contain a sequence of events, it must be possible to chain these events together while maintaining states and other properties of the FMU. Figure 4 shows the diagram layer of the rig model including the tested vehicle model. Note the inputs, both real and Boolean signals which are used to control the rig. The test rig model is built using the Vehicle Dynamics Library (Andreasson et. al., 2006) and is

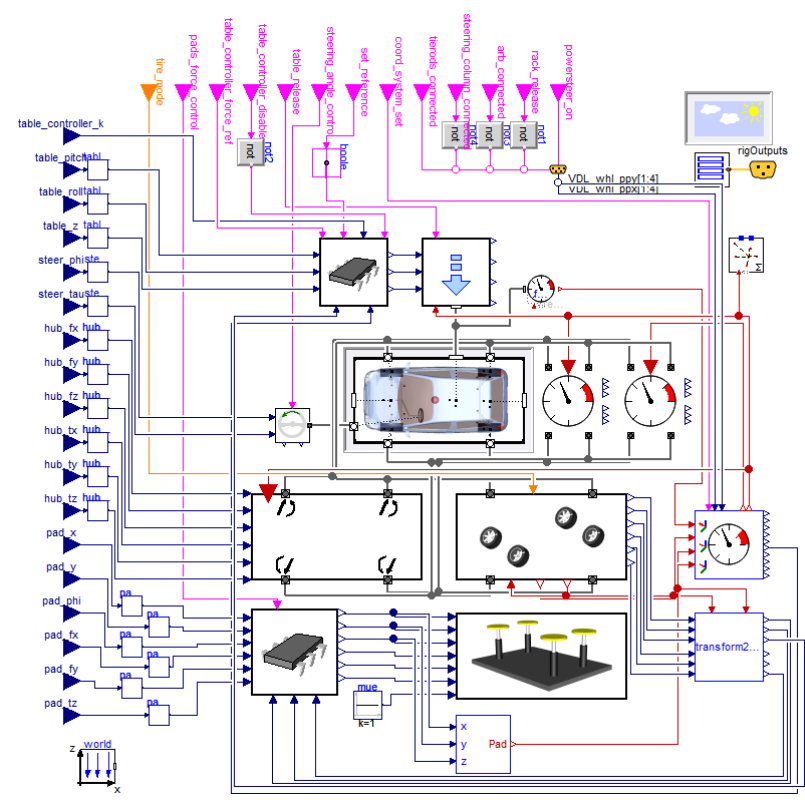

Figure 4. Diagram layer of the complete rig model.

compatible with the corresponding interface and template structure.

Each test starts with an initialization phase. Usually, the table is set to be released during this phase to let the vehicle settle. A flag coord_system_set is switched to true at an appropriate time, usually when the vehicle has settled, which then locks the vehicle coordinate system relative to the chassis and the ground coordinate system relative to ground. References for vertical load control and wheel travel are also stored for use in the test procedure. While the coord_system_set flag is set to false these coordinate systems will float in the ground plane to be centered w.r.t. to the wheelbase and track width of the vehicle. The coordinate systems are used for output signals and need to be stored after the vehicle is settled to mimic the physical procedure.

The table can then move to generate bounce, roll and pitch motion of the body relative to ground. Each of these degrees-of-freedom can be prescribed by an input signal or be controlled to maintain a fixed level of vertical load or wheel travel.

To allow these different modes to be contained in one FMU, the model is equipped with inputs that allow for each degree-of-freedom to be set independently from the others. By having these as inputs, the modes can be switched during a simulation that makes it possible to conveniently describe complex sequences as described further in Section 3.

Controllers are built to handle several different modes by using appropriate scaling parameters so a single set of controller parameters can be used for both force and position control. The control error for each wheel (force or travel) is multiplied by a coefficient contained in a $4 \times 3$ matrix which controls how the control error for each wheel affects each of the 3 table 


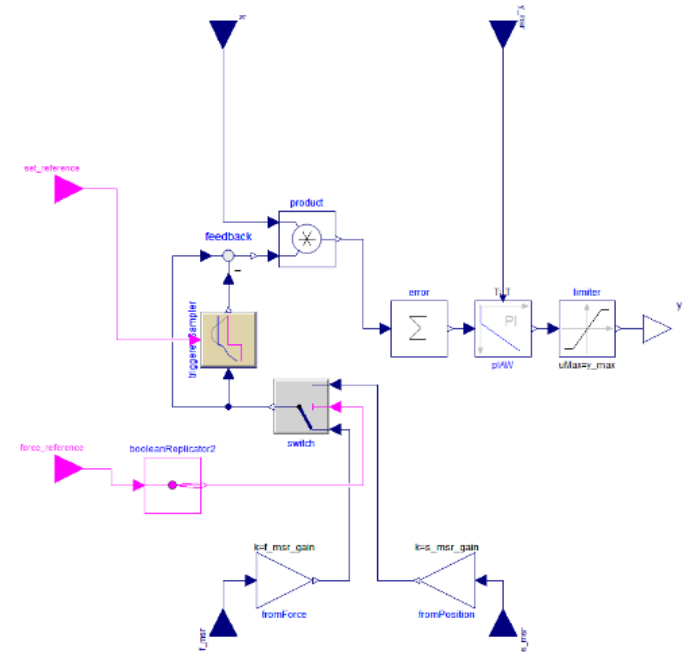

Figure 5. Controller block for one degree of freedom of the chassis table.

degrees-of-freedom. Figure 5 shows the controller block for a single degree of freedom of the chassis table. The bottom inputs are vertical load and wheel travel vectors for the wheels, each multiplied by scaling parameters. The Boolean input on the top left triggers storing of the control reference on its positive flank. The bottom left Boolean input switches between force and position control. On the top, the left input is $\mathrm{k}$, a vector corresponding to one column of the matrix mentioned above.

By setting the control parameters individually for each degree-of-freedom, it is for example possible to have a prescribed roll angle applied by the table while adjusting table bounce and pitch motion to maintain a constant total vertical load on each axle. Table 1 shows how the settings required to achieve this. The roll column has only zeroes as this is prescribed motion. The bounce column states that if load is too high, the chassis should be lifted. The pitch column state that the pitch angle should be increased if the front load is too low and/or the rear load is too high and vice versa.

The wheel pads can also be either force or position controlled. In order for the pads to be able to generate force in the tires, a tire model capable of windup in all in-plane directions is required. Typically, state-of-theart single contact point tire models do not have this capability. Therefore, a dedicated stand-still tire model is provided which acts as a linear spring-damper in the six degrees of freedom between the wheel and the pad. The model has different operating modes depending on how forces should be applied. For example, all forces and torques except pure vertical force can be disabled.

There are also inputs available to change the suspension behavior. The stabilizer and tie rods can be individually disconnected, power steering can be switch on or off and the steering rack motion can be locked.

Table 1. Table controller table for roll test with constant axle load.

\begin{tabular}{r|ccc} 
& Roll & Pitch & Bounce \\
\hline Left front & 0 & -1 & 1 \\
Right front & 0 & -1 & 1 \\
Left rear & 0 & 1 & 1 \\
Right rear & 0 & 1 & 1
\end{tabular}

\section{MATLAB Environment}

A user interface based on the MATLAB scripting language has also been developed. This interface uses functions from the FMI Toolbox for MATLAB/ Simulink to import a co-simulation FMU.

A set of standard test setups is stored in an excel spreadsheet. This spreadsheet mimics the one used for parameterizing tests on the physical test rig. There is a column for each parameter that needs to be set in the rig model and each test is defined in one row. To run a specific test, the specification for that test is read from the corresponding row in the spreadsheet based on a unique test number. The test specification is then loaded into a test object in the MATLAB environment which is sent as an argument when running the test using the test rig. The test specification can be modified after it is read from the spreadsheet by changing variables in the test

\begin{tabular}{|c|c|c|c|c|c|}
\hline Test nd & Sequence & Test Name & cycle tims & amplitude dercriptian & amplitude code \\
\hline 0 & Set-up & Set-up (vehicle initial condition) & 10 & & 0 \\
\hline 1.1 & 1.Lateral Compliand & Lateral Compliance in phase & 45 & Arytotictyrs wavticals & STVL -11 \\
\hline 1.2 & & Lateral Compliance antiphase & 45 & Hertotic tyrsurticals & STVL -11 \\
\hline 1.3 & & Lateral compliance left wheel input & 45 & Hertotic tyon unticals & STVL -11 \\
\hline 1.4 & & Lateral compliance right wheel input & 45 & thertotictyra inticals & STWL -11 \\
\hline 2.1 & 2. Longitudinal Com & Longitudinal Compliance in phase & 45 & thetotic tyrs inticalls & STVL -11 \\
\hline 2.2 & & Longitudinal Compliance anti phase & 45 & Ahetotictysenticals & STVL -11 \\
\hline 2.3 & & Longitudinal Compliance left wheel input & 45 & Artotictyrawticals & STVL -11 \\
\hline 2.4 & & Longitudinal Compliance right wheel input & 45 & Ahtotic tyon wowticals & STVL -11 \\
\hline 3.1 & 3.Âligning Torque 0 & Aligning Torque Compliance in phase & 45 & tretotictyrswaticals & STWL -11 \\
\hline
\end{tabular}

Figure 6. Excerpt from the spreadsheet containing test specifications. Each column corresponds to a parameter setting for the rig model, each row corresponds to a test. 
object. shows a small section of the test specification spreadsheet.

When comparing results from the physical rig to simulation results for $\mathrm{K} \& \mathrm{C}$ correlation work, the measurement files coming from the physical test rig can be read in and presented on the same format as is produced by the virtual test rig functions. This makes it easy to compare virtual test runs to the corresponding tests performed on the physical rig.

A typical set of commands to run a rig test from Matlab looks like this:

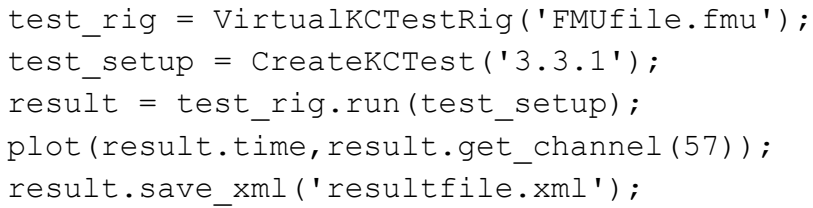

The first command, VirtualKCTestRig(), initializes the test rig object, test_rig, by loading the FMU. CreateKCTest() is then used to pull the relevant test parameters from the excel parameter sheet and store it in test_setup. The run() command of the test rig object is then called with the test setup object as an argument to run the test. The results can then be plotted directly or stored in an xml file compatible with the format of the real test rigs.

\section{Application Example}

An example of a test run in the virtual rig is shown here. The vehicle used for the example has an elastokinematic McPherson front suspension and a twist beam rear suspension with bushing mounts. During the work with the virtual rig, a new twist beam suspension model was also developed. The following plots focus on the kinematics of this rear suspension model. Parameterization is based on hard point data and other known quantities as far as possible, but some are estimated.

The test shown is a roll test with constant axle load. Figure 7 shows the roll angle measured from the physical test rig as well as the simulated roll angle in the virtual test rig. As the roll motion is directly prescribed for the test, the two signals match very well.

The vertical motion of the chassis table is used to maintain a constant total vertical load on the four wheels during the test. Figure 8 shows the measured and simulated values for the total vertical load.

The kinematics of the rear suspension for the roll test are illustrated by the following plots. Magnitudes of the signals are hidden for confidentiality reasons. Figure 9 shows the bounce motions of the two rear wheels plotted against their respective longitudinal displacement. Figure 10 shows the corresponding lateral displacement. Wheel angle, toe and camber, correlation is shown in Figures 11 and 12. The hysteresis and asymmetric behavior in the real suspension that can be seen in the measurements is not accounted for in the model which limits the accuracy that can be achieved.

Compared to the real test rig, the virtual version provides some important advantages. The user is free to change parameters in quick iterations. Depending on complexity level and length of test sequence, the execution of a complete cycle on a standard laptop normally ranges between 2 and 10s. This allow for rapid execution of DOE, and also allow for parameter tuning either to reach desired characteristics or to match with measurement data from the real test rig.

Additionally, the test rig can be used to excite any vehicle that is compatible with the standard interfaces of the Vehicle Dynamics Library, which allow the user to conveniently change vehicle configuration and model fidelity level so that the $\mathrm{K} \& \mathrm{C}$ behavior can be predicted at any time during the design process.

\section{Conclusions}

With the newly developed test rig model, it is possible to run physical and virtual test rig experiments in parallel. This simplifies correlation work since equal circumstances are ensured in both environments. Also, it facilitates moving certain tests completely to the virtual rig since specifications and output formats are equivalent. Finally, the virtual version allows for rapid iterations due to the fact that simulation and setup time is significantly less then real-time, the virtual representation allow for changes in the model that is very time consuming/expensive/impossible on real prototypes, and that simulations can be distributed onto several machines with little effort.

\section{References}

Andersson, C. (2013), A Software Framework for Implementation and Evaluation of co-Simulation Algorithms, ISBN 978-91-7473-671-7.

Andreasson, J. et. al (2006), The Vehicle Dynamics Library - Overview and Applications, in Proceedings of $5^{\text {th }}$ International Modelica Conference, Vienna, September 4-5.

Andreasson, J. et. al (2016), Deployment of highfidelity vehicle models for accurate real-time simulation, in Proceedings of First Japanese Modelica Conference, Tokyo, May 23-24.

Anthony Best Dynamics Ltd. (2014). SPMM 5000 Outline Specification - SP20016 issue 2. Wiltshire, UK.

Bastow, D. H. (2004). Car Suspensions and Handling, 4th edition. SAE International.

FMI add-in for Excel (2016) www.modelon.com/products/fmi-add-in-for-excel

Milliken, W. M. (1995). Race Car Vehicle Dynamics. SAE International. ISBN 978-1-56091-526-3 
Nilsson, T. (2013), A simulation environment for coupled systems of discontinuous ODE:s. Chapter 4, PyFMI2.0, ISSN: 1654-6229

pyFMI (2016) www.pyfmi.org

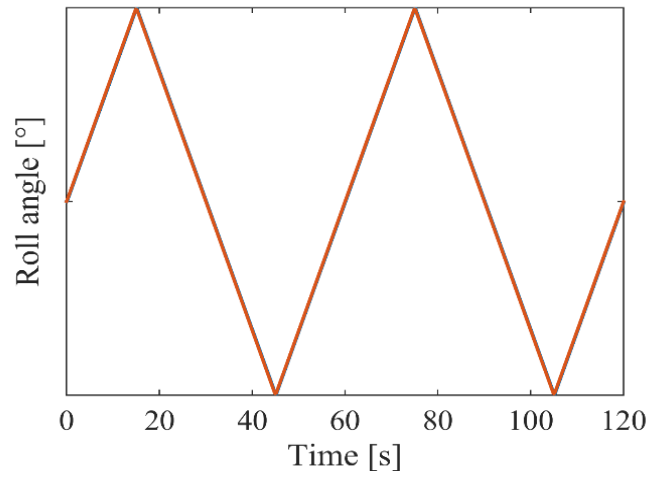

Figure 7. Roll angle, blue curve is from physical test rig, red curve is from simulation.

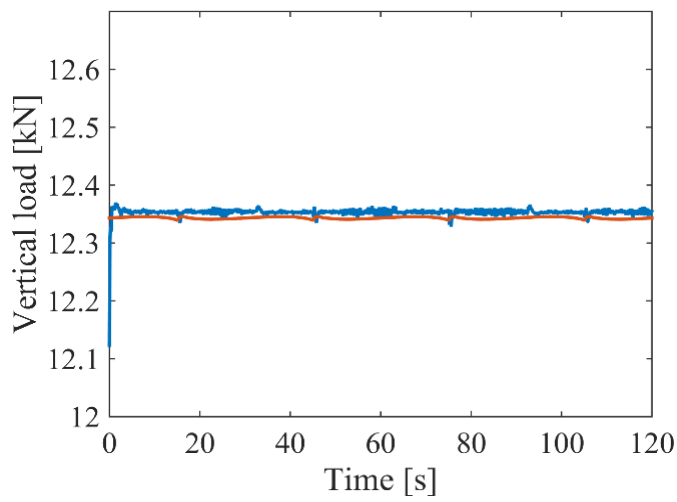

Figure 8. Total vertical force during test, blue curve is from physical test rig, red curve is from simulation.
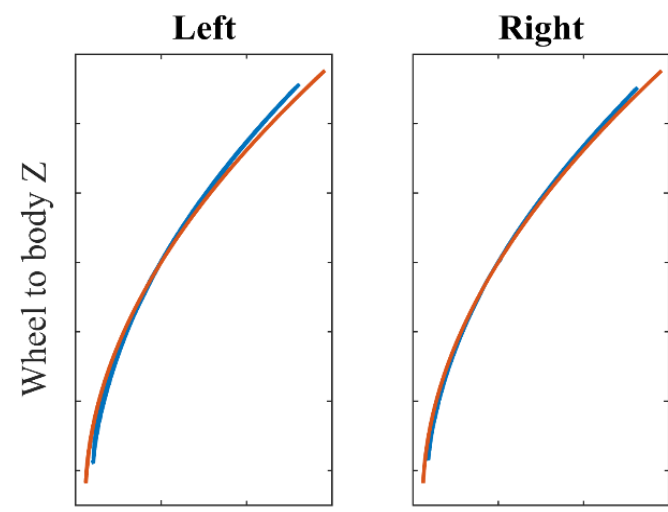

$\mathrm{X}$ displacement resolve ground

Figure 9. Vertical bounce at the two rear wheels plotted vs the corresponding longitudinal displacement, blue curve is from physical test rig, red curve is from simulation.

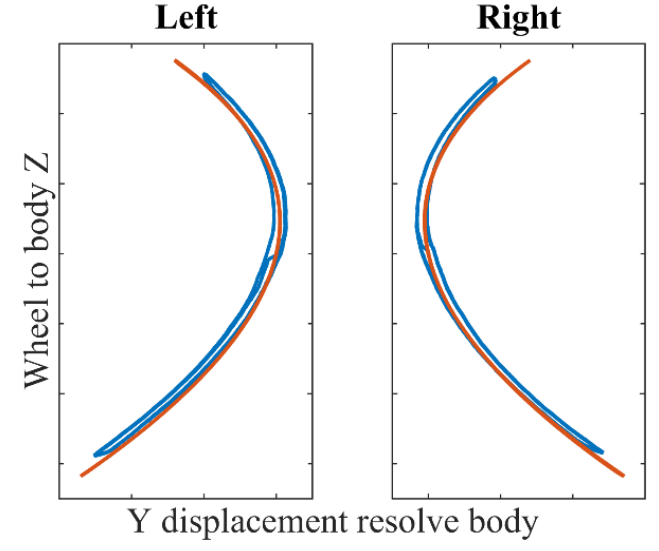

Figure 10. Vertical bounce at the two rear wheels plotted vs the corresponding lateral displacement, blue curve is from physical test rig, red curve is from simulation.

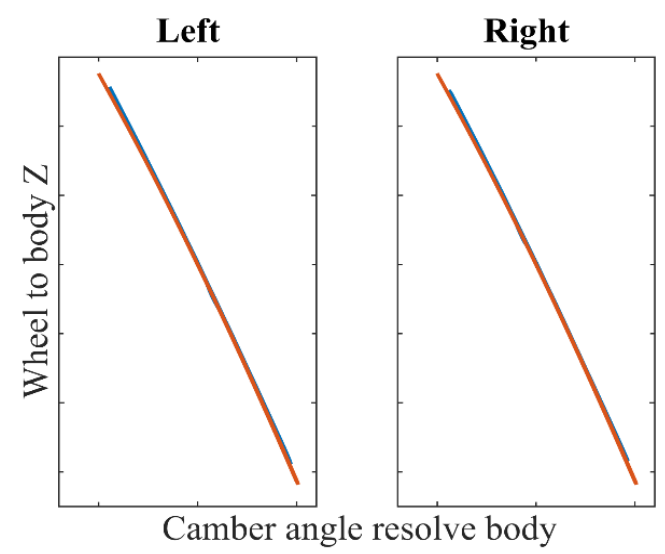

Figure 11. Camber angle at the two rear wheels plotted on the $\mathrm{x}$ axis with bounce motion on the $\mathrm{y}$ axis, blue curve is from physical test rig, red curve is from simulation.

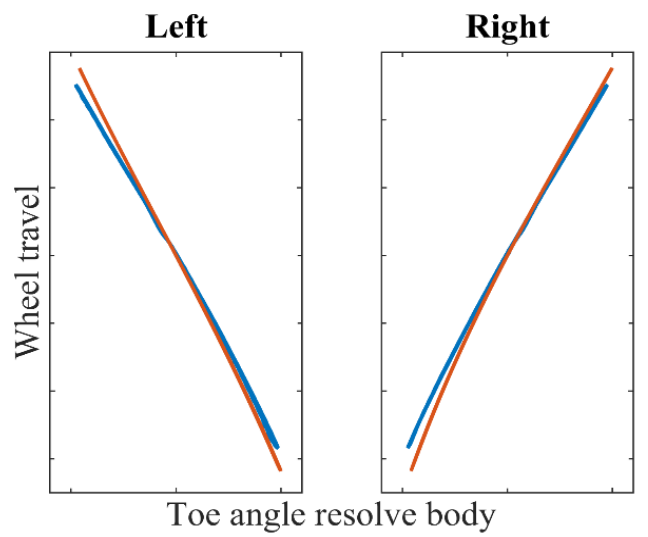

Figure 12. Toe angle at the two rear wheels plotted on the $\mathrm{x}$ axis with bounce motion on the $\mathrm{y}$ axis, blue curve is from physical test rig, red curve is from simulation. 